\title{
The Effects of Thiamine and Oxythiamine on the Survival of Cultured Brain Neurons
}

\author{
Mei-Yu Geng, Hiroshi Saito and Hiroshi Katsuki* \\ Department of Chemical Pharmacology, Faculty of Pharmaceutical Sciences, The University of Tokyo, 7-3-1 Hongo, Bunkyo-ku, Tokyo 113, Japan \\ Received January 26, 1995 Accepted May 25, 1995
}

\begin{abstract}
The effects of treatment with thiamine (Vitamin $B_{1}$ ) alone or together with its antagonist oxythiamine on the survival of brain neurons in primary culture were investigated. Treatment with thiamine significantly promoted the survival of hippocampal neurons in high cell density culture, but had no effects on the neuronal survival in low cell density culture. In addition, the survival-promoting activity exerted by thiamine was remarkably decreased by the co-application of oxythiamine, although oxythiamine used alone revealed neither a trophic nor toxic effect on the neurons of examined brain regions. The neurotrophic function of thiamine may be due to its coenzymatic role in a biochemical reaction and/or its specific function on neurotransmission and nerve conduction.
\end{abstract}

Keywords: Neuronal survival (hippocampus), Thiamine, Oxythiamine

Historically, nutritionists have been concerned with B-group vitamins for their roles in preventing diseases caused by vitamin-deficiency and for their biochemical roles as coenzymes. Rapidly accumulating evidence suggests that this is a very limited view and that vitamins are important far beyond preventing deficiency diseases. In addition, they have many other important functions independent of their coenzymatic activities (1).

Thiamine research was initiated by a common disease of the nervous system that is widespread in the world, beriberi (2). The discovery by Von Muralt and others (2) that thiamine is in some way essential to the normal function of nerve fibers had been disregarded, until other observations about the role of thiamine in the nervous system, which is different and separate from its function as a coenzyme in biochemical reactions, has been done. Evidence has been accumulating in recent years which suggest that thiamine may have a specific function in nerve conduction and transmission $(1,2)$. However, the knowledge concerning the possible role of thiamine in the development of brain neurons obtained by the techniques of cell culture has been very limited until now. Therefore, the effects of thiamine on neuronal survival were undertaken in this paper to investigate whether thiamine treatment has trophic effects on cultured brain neurons. Since it is logical to expect that an antagonist of thiamine can

*To whom correspondence should be addressed. counteract the effects exerted by thiamine, we also investigated the effects of the thiamine antagonist oxythiamine on the neuronal survival of cultured brain neurons.

Firstly, the effects of thiamine on the survival of the cultured brain neurons was examined. Procedures for cell culture and determination of neuronal survival were the same as those described in our previous paper (3). Briefly, the desired brain regions were dissected from 18-day-old embryos of Wistar rats, and cells were dissociated by digesting the tissues in $0.25 \%$ trypsin and $0.01 \%$ DNase I at $37^{\circ} \mathrm{C}$ for $30 \mathrm{~min}$. The enzymatic digestion was terminated by addition of horse serum. The tissue fragments were centrifuged at $1200 \mathrm{rpm}$ for $5 \mathrm{~min}$. The pellet was resuspended in a modified Eagle's medium supplemented with $10 \%$ fetal bovine serum. Cells were dissociated by repeated pipetting through a plastic tip with a diameter of $1 \mathrm{~mm}$. The suspension was passed through two layers of nylon mesh. Cells were seeded on polylysine-coated, plastic 48 -well plates $\left(1 \mathrm{~cm}^{2} /\right.$ well $)$ at a density of $1 \times 10^{5}$ or $5 \times 10^{3} / \mathrm{cm}^{2}$. Then cells were cultured in a humidified $\left(5 \% \mathrm{CO}_{2} / 95 \%\right.$ air) incubator. Twenty-four hours later, the medium was changed to serum-free medium, and the drugs were added at the same time. The serum-free medium is a 1:1 (v/v) mixture of Dulbecco's modified Eagle's medium (Gibco, Gaithersburg, MD, USA) and Ham's nutrient mixture F-12 (Gibco) supplemented with transferrin, insulin, progesterone and other components (for details, see ref. 1). This medium contains $6 \mu \mathrm{M}$ thiamine, 

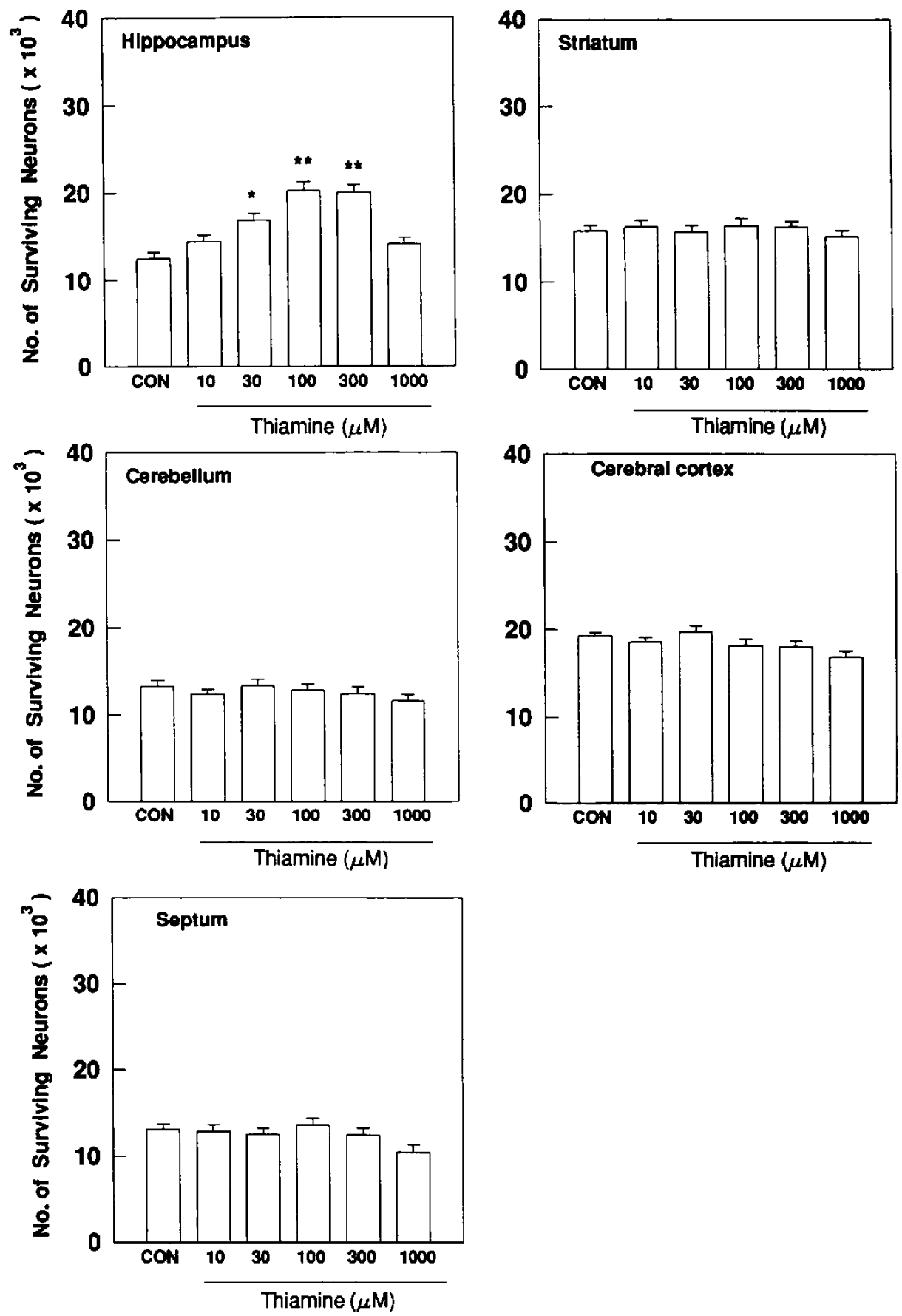

Fig. 1. Effects of thiamine on the neuronal survival of various brain regions in high cell density culture. All data are means \pm S.E.M. of 5 observations. ${ }^{*} \mathbf{P}<0.05,{ }^{* *} \mathbf{P}<0.01$ vs control group in Dunnett's test.

and the concentrations of thiamine described below are the added amount. Thiamine and oxythiamine were both generous gifts (Takeda Chemical Industries, Ltd., Osaka). Seventy-two hours later, the cultured cells were fixed with $4 \%$ paraformaldehyde and visualized by Nissl stain with Cresyl violet. The numbers of surviving neurons in each well were counted under a microscope.

Figure 1 shows the effects of thiamine on the survival of hippocampal, striatal, cerebellar, cortical and septal neurons in high cell density cultures (with the initial density of plated cells being $1 \times 10^{5} / \mathrm{cm}^{2}$ ). In the control group, less than $20 \%$ of the population of the plated neurons survived for 3 days. Treatment with thiamine significantly increased the survival of neurons only in the hippocampus at a concentration of 30,100 or $300 \mu \mathrm{M}$, with the maximal effect obtained at concentration of $100 \mu \mathrm{M}$. On the 


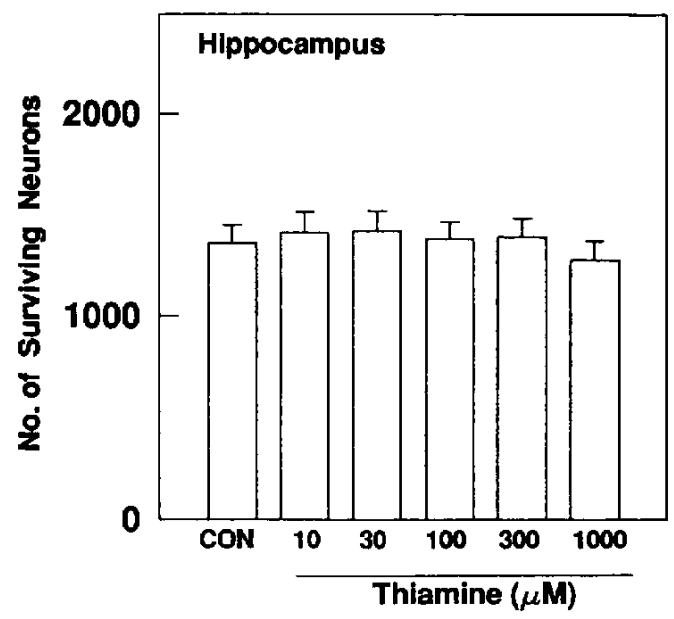

Fig. 2. Effects of thiamine on the survival of hippocampal neurons in low cell density culture. All data are means \pm S.E.M. of 5 observations.

other hand, thiamine at a lower $(10 \mu \mathrm{M})$ or higher $(1000$ $\mu \mathrm{M})$ concentration showed no effects on any of the examined brain neurons.

Figure 2 demonstrates the effect of thiamine on the survival of hippocampal neurons in low cell density culture (initial density of plated cells: $5 \times 10^{3} / \mathrm{cm}^{2}$ ). Addition of thiamine in concentrations ranging from $10 \mu \mathrm{M}$ to 1000 $\mu \mathrm{M}$ showed no significant effects on the neuronal survival.

Figure 3 shows the effects of thiamine, combined with the usage of oxythiamine, on the neuronal survival in hippocampus in high cell density culture. Oxythiamine at concentrations of $0.1 \mathrm{mM}$ and $1 \mathrm{mM}$ did not show any toxic or trophic effects on the neuronal survival in all examined brain areas (Fig. 3A). However, oxythiamine could counteract the survival-promoting effects exerted by thiamine, when applied together (Fig. 3B). The antagonizing effects held by oxythiamine were found to be concentrationdependent.

The assessment of the potential role of thiamine in neuronal function requires knowledge about the distribution of thiamine and its phosphates in the brain. Laforenza et al. (4) indicated that the level of total thiamine (sum of thiamine and its phosphoesters) is fivefold higher in neurons than in glia. The most abundant phosphoester was thiamine pyrophosphate (TPP) which accounted for $78.9 \%$ of the total thiamine in neurons and $73.4 \%$ in glia, followed by thiamine monophosphate (TMP) (12.7 and $15.1 \%$ ) and thiamine (7.5 and $11.4 \%$ ). The corresponding percentages in the intact rat brain are $84 \%$ for TPP, $7.4 \%$ for TMP and $3.5 \%$ for thiamine.

Preincubation of nervous tissue with thiamine increases the amount of TPP because thiamine is the only precursor of TPP (5). Also, thiamine pyrophosphokinase,

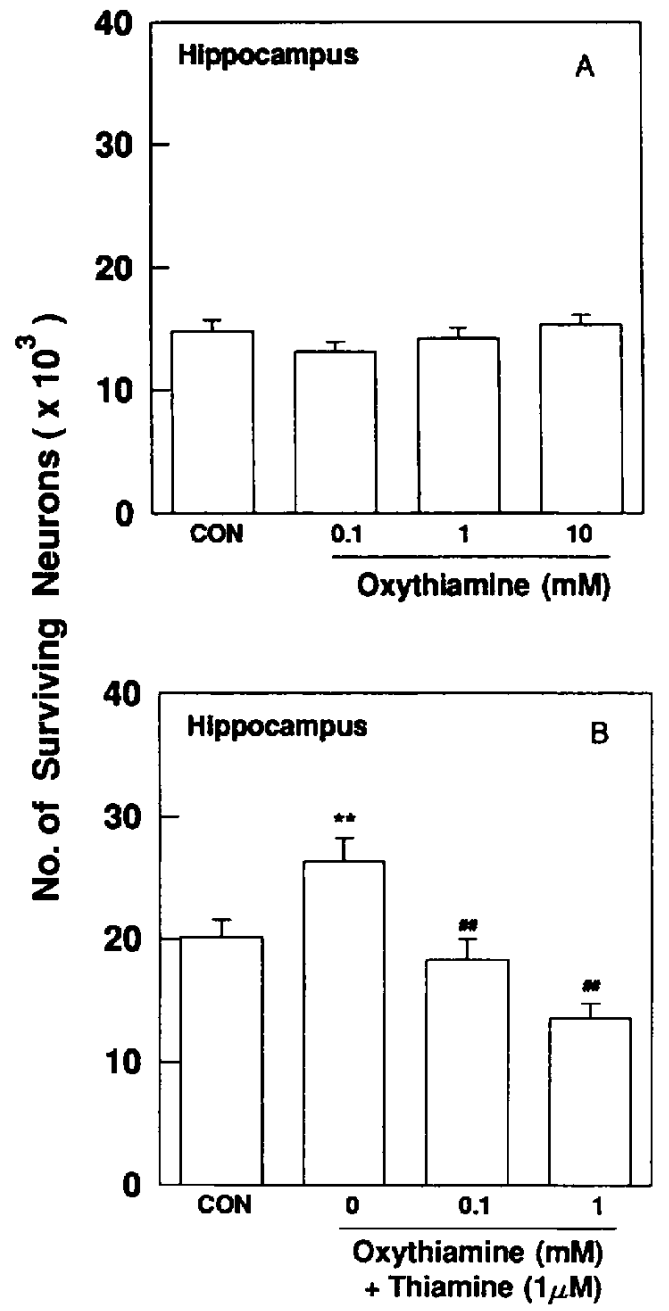

Fig. 3. Effects of treatment with oxythiamine alone and co-applied with thiamine on the survival of hippocampal neurons in high cell density culture. All the data are means \pm S.E.M. of 5 observations as shown in panels $\mathrm{A}$ and $\mathrm{B}$, respectively. ${ }^{* *} \mathrm{P}<0.01$ vs control group and ${ }^{\#{ }^{*} \mathrm{P}}<0.01$ vs thiamine-treated group in Dunnett's test.

which converts thiamine to TPP, shows higher activities in neurons than in glial and other cerebral supportive cells (5). We may hypothesize that the survival-promoting activity of thiamine in neurons is attributable to TPP. Recent evidence has highlighted the coenzymatic role of TPP in the energy metabolism of cells. It is well known that TPP acts as a cofactor of the enzymes of the tricarboxylic acid cycle in whole brain neurons. These enzymes are essential for oxidative metabolism, and their interactions with cellular calcium homeostasis have been proposed to be related to the prevention of degeneration and death of premature neurons (6). The fact that neurons are generally vulnerable to the impairment of oxidative metabolism accounts for the closed and selective relationship of these enzymes with neuronal loss $(7,8)$.

Another specific role of thiamine in the nervous system, 
independent of the known coenzymatic role of TPP, has also been postulated (9). Several lines of evidence favor the idea that the most specially neuroactive compound is thiamine triphosphate (TTP), although TTP concentrations are less than $1 \%$ of the total thiamine content (10). TTP is predominantly localized in the synaptic membrane fraction of rat brain (11) and may exert a role in the regulation of chloride permeability (9). Preincubation of a rat brain homogenate with thiamine increases the TTP content of membrane vesicles, followed by an increase in the passive uptake of ${ }^{36} \mathrm{Cl}$ by those vesicles (12). The increase in membrane conductance to $\mathrm{Cl}^{-}$may prevent excessive excitation of neurons. The preferential location of TTP in neurons over glia and the idea of a specific role of TTP in membrane excitability favor the neurotrophic function of thiamine in the nervous system.

It is also important to point out that the culture medium for neurons contains thiamine at a concentration of $6 \mu \mathrm{M}$, which is about 10 times higher than the total concentration in rat plasma (10). However, despite bathing in this thiamine-enriched enviroment, the total amount of thiamine encountered in rat hippocampus as well as in other brain regions seems rarely disturbed (10). Thus we presume that thiamine homeostasis in the brain in vivo is not only maintained at the level of the blood-brain barrier by endothelial cells but is a characteristic of nerve cells and probably of other cell types as well. The existence of such regulatory mechanisms has already been postulated (13). Our observation that neurons significantly responsed to thiamine only when it reached higher concentrations may agree with the hypothesis mentioned above.

We investigated the effects of oxythiamine, a thiamine antagonist, used alone and together with thiamine on brain neurons. Results indicated that treatment with oxythiamine together with thiamine remarkably blocked the neuronal survival-promoting activity exerted by thiamine, although oxythiamine used alone showed no effects. Since it is reported that the antagonizing effect of oxythiamine occurs after its conversion to oxythiamine pyrophosphate by thiamine pyrophosphokinase (14), we presumed that the obtained results may be attributed to the fact that the potency of oxythiamine depends on thiamine pyrophosphokinase activity (15).

Taken together, we have shown that thiamine afforded survival-promoting actions on hippocampal neurons, which were blocked by its antimetabolite oxythiamine. These findings may be related to the important role of thiamine in the nervous system, not only due to the coenzymatic role of TPP in biochemical reactions but also due to the specific neuroactive action of TTP in nerve conduction and transmission. These interesting effects suggest that thiamine may have potential use in the therapy against neurodegenerative diseases. Further investiga- tions must be made to determine why thiamine influenced the hippocampal neurons only in high density culture. In addition, the detailed mechanisms by which oxythiamine exerted its antagonizing effect after co-application with thiamine remain to be clarified further at the molecular level.

\section{REFERENCES}

1 Howerde ES and Lawrence JM: Beyond deficiency: New views on the function and health effects of vitamins. Ann NY Acad Sci 669, 1-404 (1992)

2 Henry ZS and Clark JG: Thiamine and twenty years of progress. Ann NY Acad Sci 378, 1-470 (1982)

3 Okuda S, Saito $\mathrm{H}$ and Katsuki H: Arachidonic acid: toxic and trophic effects on cultured hippocampal neurons. Neuroscience 63, 691-699 (1994)

4 Laforenza U, Patrini C and Rindi G: Distribution of thiamine, thiamine phosphates and thiamine metabolizing enzymes in neurons and glial cells enriched fractions of rat brain. J Neurochem 51, 730-735 (1988)

5 Rindi G, Comincioli V, Reggiani $C$ and Patrini C: Nervous tissue thiamine metabolism in vivo. II. Thiamine and its phosphoesters dynamics in different brain regions and sciatic nerve of the rat. Brain Res 293, 329-342 (1984)

6 Gibson GE, Ksiezak RH and Sheu KFR: Correction of enzymatic, metabolic and behavioral deficits in thiamine deficiency and its reversal. Neurochem Res 9, 803-814 (1984)

7 Gibson GE, Sheu KFR, Blass JP, Baker A, Carson KC, Harding B and Perrino P: Reduced activities of thiamine-dependent enzymes in the brain and peripheral tissues of patients with Alzheimer's disease. Arch Neurol 45, 836-840 (1988)

8 Butterworth RF and Besnard AM: Evidence for defective thiamine neurochemistry in Alzheimer's disease. J Cerebr Blood Flow and Metab 11, S21 (1991)

9 Bettendorff L, Hennuy B, Wins P and Schoffeniels E: Thiamine and derivatives as modulators of rat brain chloride channels. Neuroscience 52, 1009-1017 (1993)

10 Bettendorff $L$, Weekers $L$, Wins $P$ and Schoffeniels E: Injection of sulbutiamine induces an increase in thiamine triphosphate in rat tissues. Biochem Pharmacol 40, 2557-2560 (1990)

11 Matsuda T, Tonomura H, Baba A and Iwata H: Tissue difference in cellular localization of thiamine phosphate esters. Comp Biochem Physiol 94B, 405-409 (1989)

12 Bettendorff $L$, Wins $P$ and Schoffeniels E: Regulation of ion uptake in membrane vesicles from rat brain by thiamine compounds. Biochem Biophys Res Commun 171, 1137-1144 (1990)

13 Bettendorff $L$, Peeters $M$, Jouan C, Wins $P$ and Schoffeniels E: Determination of thiamine and its phosphate esters in cultured neurons and astrocytes using an ion-pair reversed-phase highperformance liquid chromatographic method. Anal Biochem 198, 52-59 (1991)

14 Eusebi AJ and Cerecedo LR: In vivo and in vitro studies with oxythiamine and neopyrithiamine. Fed Proc 9, 169-173 (1950)

15 Nishimura H, Kawasaki $Y$, Nosaka $K$, Kaneko $Y$ and Iwashima A: A constitutive thiamine metabolism mutation, thi80, causing reduced thiamine pyrophosphokinase activity in Saccharomyces cerevisiae. J Bacteriol 173, 2716-2719 (1991) 